\title{
La implementación de metodologías activas de enseñanza-aprendizaje en educación superior para el desarrollo de las competencias genéricas de innovación y comunicación en los primeros años de Ingeniería
}

\section{The implementation of active teaching and learning methodologies in higher education for developing generic competences of innovation and communication in the first years of an engineering program education}

Recibido: 6 de abril de 2019 | Aprobado: 26 de mayo de 2019

\section{Resumen}

En este trabajo se presenta un ciclo de investigación-acción referido a la implementación de metodologías activas de enseñanza-aprendizaje para el desarrollo de las competencias genéricas de comunicación e innovación en un curso de carácter basal en la Educación en Ingeniería denominado 'Introducción a la Ingeniería' en el plan de estudios de una universidad chilena. El objetivo de esta investigación es presentar una propuesta de implementación de recursos y estrategias para un aprendizaje profundo y la evaluación de competencias genéricas en los primeros años de ingeniería y ciencias. Para ello, se realiza un trabajo colaborativo e interdisciplinario entre ingenieros-docentes de la facultad y las unidades de apoyo a la docencia y al aprendizaje en el área de STEM (ciencias, tecnología, ingeniería y matemáticas por sus siglas en inglés) bajo el paradigma de la investigación-acción con foco en un plan de mejoramiento innovado con los lineamientos de la metodología de Educación en Ingeniería CDIO (UCSC, 2014) Concebir - Diseñar - Implementar - Operar (Lopera y Restrepo, 2015), añadiendo dos componentes principales: (1) evaluación y (2) enseñanza de la innovación. Los principales resultados muestran un alto grado de satisfacción positiva por parte de los estudiantes en relación con la implementación de nuevas estrategias de enseñanza y de procedimientos evaluativos. Se releva también el trabajo en colaboración realizado por un equipo multidisciplinario de

\footnotetext{
* Candidato a Doctor en Lingüística Cátedra Unesco para la Lectura y Escritura (PUCV). Magíster en Letras con mención en Lingüística, Profesor de Castellano y Licenciado en Letras y Educación por la Pontificia Universidad Católica de Chile. Es Coordinador del Programa de Alfabetización Avanzada y Director del Laboratorio de Escritura Armadillo FCFM de la Facultad de Ciencias Físicas y Matemáticas de la Universidad de Chile, Chile. Para contactar al autor: enrique.sologuren@uchile.cl

** Magíster en Lingüística Aplicada y Profesora de Castellano por la Pontificia Universidad Católica de Valparaíso, Chile. Es asesora Pedagógica del Programa de Alfabetización Avanzada y tutora especialista en el Laboratorio de Escritura Armadillo FCFM de la Facultad de Ciencias Físicas y Matemáticas de la Universidad de Chile, Chile. Para contactar a la autora: carmen.nunez@uchile.cl

*** Doctora en Didáctica y evaluación educativas por la Universidad de Alcalá de Henares, España. Académica de la Facultad de Educación de la Universidad Bernardo O'Higgins, Santiago de Chile. Para contactar a la autora: isagonzalez67@gmail.com
}

ISSN (en línea): 1814-4152 / Sitio web: http://cuaderno.pucmm.edu.do

CÓMO CITAR: Sologuren, E., Núñez, C. y González, M. (2019). La implementación de metodologías activas de enseñanza-aprendizaje en educación superior para el desarrollo de las competencias genéricas de innovación y comunicación en los primeros años de Ingeniería. Cuaderno de Pedagogía Universitaria, Vol. 16, n. ${ }^{\circ}$ 32, julio-diciembre, pp. 19-34 
profesionales y la relevancia asignada al desarrollo progresivo de las competencias genéricas de comunicación e innovación. Adicionalmente, se analizan los nudos críticos que profesores y estudiantes manifiestan en su proceso de aprendizaje e inserción a la disciplina. Finalmente, se muestran las implicancias para el desarrollo de las competencias genéricas a través del curriculum.

Palabras clave: Estrategias metodológicas de enseñanza; evaluación; competencia de comunicación e innovación en el área de STEM; formación transversal; educación en ingeniería y ciencias.

\section{Abstract}

This article presents an action-research cycle about the implementation of active teaching and learning methodologies for developing generic competences of generic competences of communication and innovation in a core course in Engineering Education called 'Introduction to Engineering' as part of a studies program taught in a Chilean university. The aim of this research is to design a proposal of resources and strategies for developing deep learning and the evaluation of generic competences in the first years of Engineering and Science education. For this, a collaborative and interdisciplinary work between engineers and professors of the faculty and the teaching and learning support units in the areas of STEM (Science, Technology, Engineering and Mathematics fields) was conducted under the action-research paradigm focused on an improvement plan innovated with quidelines of the methodology of Engineering Education CDIO (UCSC, 2014) Conceive - Design - Implement - Operate (Lopera y Restrepo 2015), adding two primary components: (1) evaluation and (2) teaching of innovation. The main results show a high level of positive satisfaction of the students in relation to implementing new teaching and evaluative methods strategies. The collaborative work conducted by a multidisciplinary professional team is outlined, as well as the relevance assigned to the progressive development of generic competences of communication and innovation.

Keywords: Methodological strategies of teaching; evaluation; communication and innovation competence in STEM fields; transversal training, engineering Education.

\section{Introducción}

Este trabajo da cuenta de una investigación-acción en el ámbito de la implementación de estrategias y recursos para desarrollar las competencias genéricas en un curso de carácter basal en la Educación en Ingeniería denominado 'Introducción a la Ingeniería' en el plan de estudios de una universidad chilena. Este espacio curricular tiene como objetivo principal que los estudiantes desarrollen proyectos de diseño en Ingeniería, para así promover la creatividad y fortalecer su motivación intrínseca por el aprendizaje de las disciplinas STEM (ciencias, tecnología, ingeniería y matemáticas por sus siglas en inglés). Para lograr esto, el curso propone la aplicación de los conocimientos básicos a la solución de problemas de ingeniería en un marco ético de actuación y desempeño académico. De esta manera, este espacio permite desplegar habilidades socioemocionales en el estudiantado y conjugarlas con los conocimientos técnicos en ciencias de la ingeniería.

El propósito de este ciclo de investigación-acción es diseñar una propuesta de recursos y estrategias de enseñanza-aprendizaje para el desarrollo de competencias genéricas en los primeros años de ingeniería y ciencias. Nuestra pregunta de investigación es: ¿Cómo contribuir hacia un modelo de aprendizaje activo del curso de Introducción a la Ingeniería que fortalezca el desarrollo en competencias genéricas? Específicamente, este estudio aplicado considera los siguientes componentes axiales: primero, la innovación en las metodologías docentes. Segundo, el desarrollo y progresión de dos competencias genéricas o transversales clave: la innovación y la comunicación efectiva en lengua materna. Tercero, la reflexión pedagógica al interior de los equipos docentes. Estos tres pilares sustentan una propuesta que permite mejorar y fortalecer los procesos de evaluación de aprendizaje en el primer ciclo formativo: plan común de ingeniería y ciencias.

Para cumplir con este objetivo, el plan de mejoramiento innovado enriquece la metodología de Educación en Ingeniería CDIO Concebir - Diseñar - Implementar - Operar (Lopera y Restrepo, 2015), añadiendo dos componentes principales: (1) enseñanza de la comunicación efectiva y (2) enseñanza de la innovación. Esto implica un trabajo colaborativo e interdisciplinario entre ingenieros-docentes de la Escuela de Ingeniería y 
Ciencias y las unidades de apoyo a la docencia y al aprendizaje en el área de STEM.

De esta manera, el trabajo conjunto entre diferentes actores - docentes, especialistas en Educación y en competencias genéricas levantó un plan de innovación del proceso de enseñanza-aprendizaje para el primer ciclo de ingeniería y ciencias. Este plan contempla la aplicación de una diversidad de metodologías activas de enseñanza-aprendizaje basadas en TIC y la creación de instrumentos de evaluación pertinentes para el monitoreo y progresión de las competencias genéricas.

El presente artículo se organiza del siguiente modo: para comenzar, se contextualiza la propuesta y se explicitan sus fundamentos teóricos. Luego, se presenta la metodología y sus fases de trabajo. Posteriormente, se desarrollan las principales estrategias metodológicas y evaluativas implementadas en el curso, así como los principales resultados obtenidos. Finalmente, se ofrecen algunas reflexiones y se trazan nuevos ciclos de investigación-acción que pueden ser explorados.

\section{Marco de referencia}

\section{Explorando el macrocurriculum: la noción de competencia y el desarrollo de competencias genéricas}

Una competencia se define como la instancia donde una realidad compleja exige seleccionar entre el universo de conocimientos, capacidades y habilidades aquellos aprendizajes conceptuales, procedimentales y actitudinales que se requieren para la comprensión y transformación de esta realidad (Wehlburg, 2014). Su desarrollo en el individuo exige no solo capacidad de gestión global de las mismas, sino que también un cierto grado de conjunción con determinadas actitudes y valores personales. La figura $n^{\circ} 1$ expresa la relación de subconjunto de los conocimientos, habilidades, destrezas y capacidades respecto de las competencias.

Las competencias genéricas o transversales por su parte se refieren a las habilidades y conductas que dan cuenta de los desempeños superiores o relevantes en el ámbito laboral y profesional (Le Boterf, 2011; González, 2016); y que, por lo regular, se expresan verbalmente en términos de atributos o rasgos personales, como resultan ser los casos de la orientación al logro, la proactividad,

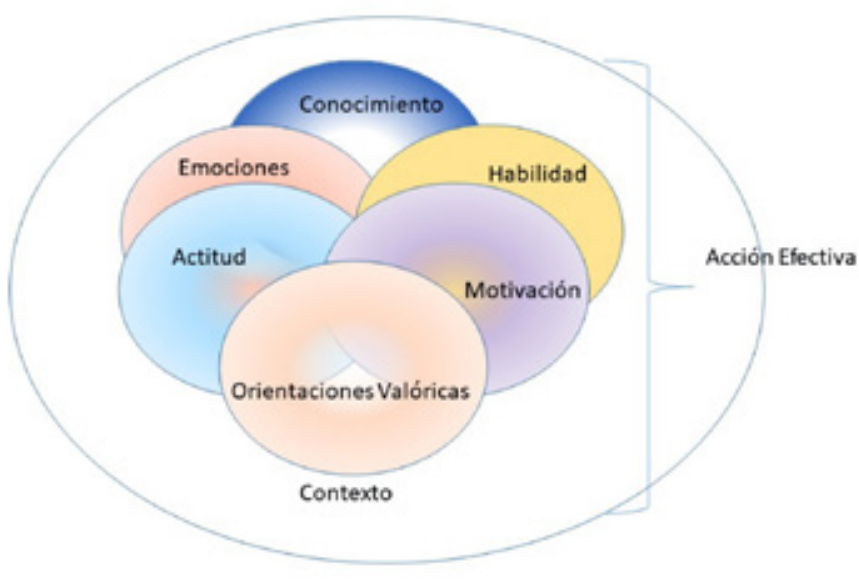

Figura 7: Componentes involucrados en la definición de competencia (González, 2016)

la rigurosidad, la flexibilidad, la comunicación efectiva y la innovación.

En la Facultad de Ciencias Físicas y Matemáticas de la Universidad de Chile (FCFM-UCH), estas competencias son declaradas en los planes de estudio y se encuentran graduadas en sendos mapas de progresión (Uribe, Sologuren y Matamoros, 2016). En la Tabla 1 se detalla el nivel de logro establecido para el primer ciclo formativo de Ingeniería y Ciencias.

Así las cosas, fortalecer estas habilidades transversales configura un aprendizaje a lo largo de la vida que se desarrolla de forma situada, en forma específica en las diferentes disciplinas y subdisciplinas de la ingeniería. Por lo tanto, se trata de competencias que no se desarrollaron de una vez y para siempre, necesitan ponerse en práctica en escenarios específicos, ricos en oportunidades de aprendizaje y desafiantes para la promoción de la innovación, la multidisciplina y el emprendimiento.

Los trabajos que identifican habilidades de innovación coinciden en señalar las habilidades comunicativas como una de las destrezas que siempre está presente en el perfil de un innovador. Cobo (2013, p. 10) la define como: "comunicar la información y las ideas de manera efectiva a las múltiples audiencias, usando una variedad de formatos". Asimismo, afirma que esta habilidad junto con las otras (colaboración, creatividad, pensamiento crítico, aprendizaje contextual, etc.) son fundamentales para lograr el aumento de la productividad. 
Tabla 7: Nivel de logro de las competencias genéricas de Comunicación (CG1) y de Innovación (CG6) según el mapa de progresión de la facultad (FCFM, 2018)

\begin{tabular}{|l|l|}
\hline $\begin{array}{c}\text { COMPETENCIA DE COMUNICACIÓN ACADÉMICA Y PROFESIONAL } \\
\text { (CG1) }\end{array}$ & \multicolumn{1}{c|}{$\begin{array}{c}\text { COMPETENCIA DE INNOVACIÓN } \\
\text { (CG6) }\end{array}$} \\
\hline $\begin{array}{l}\text { "Leer y escuchar de forma analítica diferentes tipos de textos pertinentes } \\
\text { para su formación. Asimismo, expresar de manera eficaz, clara e infor- } \\
\text { mada sus ideas, en situaciones académicas formales, tanto en modalidad } \\
\text { oral como escrita, en español" (FCFM, 2018). }\end{array}$ & $\begin{array}{l}\text { "Demostrar pensamiento asociativo al observar, cuestionar y } \\
\text { explorar alternativas, valorando el conocimiento distinto al pro- } \\
\text { pio como fuente válida para generar procesos de búsqueda y } \\
\text { descubrimiento de soluciones novedosas a problemas o nece- } \\
\text { sidades" (FCFM, 2018). }\end{array}$ \\
\hline
\end{tabular}

Es más, algunos autores han comenzado a configurar el concepto de "Alfabetización en innovación" (Innovation Literacy), que se define como: "[...] the capacity of an individual to understand and use written text and/or graphs to make well-founded judgments and scientific inferences about processes and procedures with the goal of collaboratively constructing a new original product" / "La habilidad de una persona de entender y utilizar textos escritos y/o gráficos para producir opiniones y conclusiones científicas sobre procesos y métodos con el fin de construir un producto nuevo y original de forma colaborativa" (Erdogan, Sencer y Capraro, 2013, p. 3). El modelo educativo de la Universidad de Chile declara el desarrollo de las competencias genéricas-sello con el objeto de apuntar a la formación de excelencias con un aumento sostenido y permanente de la calidad, la equidad y la pertinencia del pregrado (UCHILE, 2018), y en este sentido, el desarrollo de estas competencias genéricas apunta a la profundización de las dimensiones académicas y profesionales.

\section{Accediendo al microcurriculum: la retroalimentación en el aula de ingeniería}

La retroalimentación es una pieza clave y constitutiva de la evaluación auténtica; entendida esta última como un proceso que contribuye a la autonomía de los que intervienen en el proceso de enseñanza-aprendizaje y a su mejora y transformación (Margalef, 2005). Por lo tanto, la evaluación se convierte en un proceso de investigación que no queda reducido solo a la calificación de los alumnos (Ahumada, 2005). Supone, entonces, una valoración de todo el proceso y de todos sus participantes, utilizando para ello una variedad de estrategias y procedimientos.

Además, la evaluación auténtica no es vista como una cuestión más que tenemos que hacer, sino una decisión consciente y fundamentada (Álvarez, 2001, en Margalef, 2005). Ella está centrada mayoritariamente en procesos más que en resultados y pone énfasis en que el estudiantado asuma un rol protagónico en su propio aprendizaje, en el que la evaluación (y la retroalimentación) sea un medio que le permita alcanzar los conocimientos propuestos de su formación universitaria.

En esta concepción de evaluación auténtica el feedback o retroalimentación es un componente pivotal de esta propuesta. Camilloni (2009) señala que, si el docente logra centrar su atención en tratar de comprender qué y cómo están aprendiendo sus alumnos, en lugar de concentrarse en lo que les enseña, se abre la posibilidad de que la evaluación deje de ser solo un modo de constatar el grado en que los estudiantes han captado correctamente el contenido, ampliando la visión a cómo han logrado desarrollar las competencias pretendidas. Se considera, entonces, como retroalimentación el momento en que el profesor evalúa a sus estudiantes y les detalla las actividades a las que han de dedicar más esfuerzo para luego proceder eventualmente al reconocimiento.

Un elemento fundamental de la retroalimentación en la evaluación por competencias es su condición cíclica (Brown, 2015; Coll, Rochera, Mayordomo y Naranjo, 2011). Ella se efectúa continuamente en el instante en que el/la evaluador(a) opta por hacer algunas pausas para llevar a efecto tales tareas.

En este sentido, la retroalimentación se entiende como un contrato, implícito o explícito, que se acuerda en las primeras sesiones del curso, momento en que se acepta aquello de lo que se hará responsable el profesor, por un lado, y los alumnos, por otro. Asimismo, se negocian la planificación general del curso, tanto en lo relacionado al discurso temático como al discurso metodológico y la modalidad de evaluación propuesta. 
El docente, cuando planifica su curso, implícitamente está pensando en cómo evaluar el proceso de aprendizaje del estudiante. Una vez que visualiza qué espera de ellos(as), provocará ciertas actividades que se evidencian en aquellas estrategias de enseñanza que presentará a sus alumnos. Esto tiene el objetivo de desarrollar en ellos algunas competencias propias del curso y/o del perfil de egreso de la carrera en cuestión. El proceso de enseñanza es, por tanto, clave para el desarrollo del aprendizaje del estudiante (Beneitone, Esquetini, González, Maletá, Suifi y Wagenaar, 2007; Zabalza, 2014). El desempeño del docente, a su vez, se evidenciará en su evaluación según criterios establecidos en su unidad académica o a nivel institucional. Es notorio, por tanto, la interdependencia evaluativa docente-alumno.

Margalef y Canabal (2010, p. 20) sostienen que "la retroalimentación continua y la toma de conciencia sobre la práctica y lo aprendido son oportunidades que debemos aprovechar, así como la generación de vínculos más cercanos entre docentes y estudiantes, ya que cuanto mayor sea la relación, mayor será el aprendizaje por ambas partes". De esta cita se desprende, además de la relevancia de la noción de retroalimentación, el rol que juega el docente en el desarrollo de competencias genéricas y específicas. A esto se suma el desarrollo en un ámbito particular: el ingenieril, disciplina que en el mundo globalizado de hoy reconoce las dimensiones antropológicas y pragmáticas en la identidad profesional de ingeniero (Breeze y Sancho Guinda, 2017) necesarias para la comunicación y la innovación a través de las culturas.

\section{Buenas prácticas del profesorado} universitario: la base necesaria para implementar metodologías activas de enseñanza-aprendizaje

Un desarrollo de competencias y su debida evaluación es posible en la medida en que los profesores se comprometen con su desarrollo profesional docente. Es decir, no se trata de implementar un currículo y un modelo educativo, sino en que mejore el aprendizaje y nuestros estudiantes se vuelvan competentes para la vida. Esto será posible en la medida en que los maestros se comprometan en la mejoría de su práctica. A este respecto, Zabalza (2014), a partir del análisis de una cantidad importante de investigaciones sobre lo que hacen los mejores profesores universitarios, sintetiza las principales características de una buena práctica docente (Tabla 2).

Todos estos elementos son requeridos entre los profesionales que ejercen en el ciclo formativo inicial o Plan Común de Ingeniería y Ciencias. De igual forma, desde un enfoque centrado en la investigación de la profesión docente, Contreras (1995) señala que la labor docente no se limita a la puesta en práctica de planes y programas elaborados por otros, sino que, a la inversa, la realización de la tarea de enseñanza necesita tanto de un cierto dominio de habilidades técnicas y recursos para la acción, como de un conocimiento de la cultura y de las disciplinas que constituyen el ámbito o el objeto de lo que se enseña.

Entonces, el profesional universitario, en este contexto, también necesita desplegar competencias profesionales complejas que implican la toma de decisiones, desarrollo de habilidades y principios.

Tabla 2: Principales características de una buena práctica docente según Zabalza (2014)

1) Hacer visibles los patrones de análisis y los enfoques que los responsables educativos y el profesorado u otros agentes implicados utilizan a la hora de definir y describir su actuación y dar sentido a su propia práctica.

2) Mejorar los dispositivos metodológicos y técnicos para el análisis de prácticas educativas.

3) Mejorar los dispositivos narrativos y gráficos para visibilizar mediante esquemas conceptuales y ejes de identificación de los núcleos sustanciales, conceptuales y operativos, en torno a los cuales el profesorado y los responsables describen y valoran sus propuestas educativas.

4) Ofrecer un valioso bagaje de conocimientos y experiencias, profundo pero manejable, capaz de suscitar el debate dentro de la comunidad científica y profesional vinculada a ese nivel educativo en torno a las estrategias más adecuadas para mejorar la calidad de las propuestas que se hagan para responder a las demandas de los diferentes contextos culturales y sociales.

5) Identificar las características propias de la buena práctica estudiada destacando en ella aquello que pudiera servir de modelo de actuación en el ámbito educativo al que pertenezca. 
Ha de tener conciencia del sentido y de las consecuencias de su práctica. La actividad del docente no se reduce, entonces, a una actividad técnica y previsible, pues exige la resolución de múltiples y variadas situaciones, caracterizadas por un alto grado de incertidumbre. Además, el saber hacer del docente se construye y enriquece tanto en la experiencia individual como en la colectiva, ambas en un proceso de progresivas reelaboraciones. Aquello que lo hace competente profesionalmente tiene relación tanto con el caudal de conocimiento de que dispone como con los recursos intelectuales que posibilitan el incremento y desarrollo del conocimiento profesional. En palabras de Imbernón (2012, p. 101), hay que "repensar la práctica docente desde la conciencia de la contextualización y la complejidad del acto educativo".

En esa línea, Perrenoud (2003) indica que una definición de competencia debe considerar la incorporación a la enseñanza de nuevas tecnologías, el incremento de las tareas de diseño y la demanda de trabajo en equipo. Enfatiza que los grados de complejidad y de diversificación de la labor docente no implica dejar de lado las usuales prácticas como la planificación, sino su consideración en un ámbito más complejo, aunque también más rico. Por último, es necesario poner de manifiesto que las cualidades particulares de la formación técnico-profesional emanan fundamentalmente del propósito de esta clase de formación: preparar para el trabajo y el mundo profesional.

Arnold (2002) señala esta determinación, estableciendo una escisión entre los fines de la pedagogía y los de la formación profesional, esta última definida como "ciencia del desarrollo de las competencias". Por tanto, los aspectos relevantes para el desempeño del profesorado reflexivo, según este mismo autor, tienen relación con capacidades para vincular férreamente la formación a las cualidades de la actividad profesional. Estos aspectos ponen de manifiesto que cuando hablamos de formación para el trabajo, se hace imperativo procurar la integración de los saberes específicos y pedagógicos, pues los segundos no se hacen suficientes y tampoco son independientes de los contenidos y competencias a desarrollar. Con estos elementos podemos tener una primera imagen de las cualidades que debe incluir el perfil del profesional integral.

\section{La propuesta de investigación y acción}

\section{Contexto e insumos curriculares}

Un aspecto fundamental del proceso de innovación curricular de la formación profesional de ingenieros(as) y científicos(as), radica en el cambio de la cultura formativa del estudiante de la Escuela de Ingeniería y Ciencias. Si bien es cierto que este cambio posee altos niveles de consenso por su impacto en la creación del estilo de aprendizaje profundo, persisten igualmente tensiones en el sistema educativo como la permanencia de un modelo de desempeño tradicional que favorece procedimientos evaluativos estandarizados. A esto se suman las necesidades de formación docente de los académicos(as).

En este sentido, es necesario revisar el modelo evaluativo del profesor, quien es tanto creador del curriculum como intérprete de este. Como intérprete del currículum, el académico debe examinar sus propias prácticas metodológicas y evaluativas, para determinar su impacto en la formación inicial de los estudiantes, considerando posibles inconsistencias en el modelo evaluativo. En este ciclo de investigación-acción nos basamos en la evaluación auténtica (Brown, 2015), por medio de implementación de un plan de mejoramiento de la enseñanza innovado en la enseñanza superior. También nos propusimos transitar desde el conocimiento fragmentado, parcelado con escasa interdisciplinaridad de los tradicionales planes de estudios universitarios hacia una renovación de la oferta académica en curso. Por ello, el modelo educativo y el perfil de los titulados es un elemento clave a revisar. Asimismo, los conocimientos que adquieran, su capacidad de aplicarlos y las competencias, destrezas y actitudes con que terminen su período de formación debe ser una cuestión fundamental que debe plantearse en la planificación académica y en el seguimiento de los resultados alcanzados (Michavila, 2009).

\section{Reflexión docente}

La reflexión docente es otro componente esencial en los procesos de innovación curricular. El quehacer profesional del docente, al igual que otras actividades profesionales, necesita para su desempeño de competencias reflexivas. Competencia fundamental en el mundo actual, ya que es difícil para los académicos y profesionales contar con una 
batería de respuestas para las múltiples situaciones propias de su labor y, en la mayoría de los casos, se torna imprescindible combinar conocimientos y experiencias anteriores, para poder poner en marcha una respuesta idónea. En este sentido, Perrenoud (2007) habla de la práctica reflexiva, lo que implica "la reflexión en la acción" y "la reflexión sobre la acción". Esto significa que el académico o profesional produce nuevo conocimiento respecto de la enseñanza a partir de la práctica. Ello supone que, desde la experiencia y la capacidad reflexiva, el docente genera nuevas respuestas a los problemas que sugieren su propia labor.

En la misma línea, puede afirmarse que se trata de un profesional que no sólo implementa el currículum, sino que lo interpreta, que recrea cada situación de manera vivencial de acuerdo con los requerimientos de los estudiantes, desarrollando en estos, de modo más concreto, las competencias comprometidas en el perfil de egreso de la carrera.

\section{Competencias y formación integral}

Las habilidades comprometidas a evaluar en el curso Introducción a la Ingeniería tienen la finalidad de desarrollar:

\section{1.- Resolución de problemas \\ 2.- Creatividad \\ 3.- Trabajo en equipo \\ 4.- Comunicación efectiva}

Para este estudio se abordarán el desarrollo de las Competencias Genéricas (FCFM, 2018):

\section{1.- Innovación \\ 2.- Comunicación académica y profesional}

La totalidad de estas habilidades se orienta a una formación integral del estudiante, de modo que no se acote solamente a la enseñanza y el aprendizaje de conocimientos científicos, tecnológicos y a la aplicación de estos, sino que incluya, también, una educación de orden humanista que actúe como preparación para la vida. Este proceso supone considerar todos aquellos componentes que se necesiten en la evaluación, como la comprensión de su relación interna y el significado de cada uno de ellos. Cada faceta de este conjunto de fases precisa de un tiempo determinado para que se suscite un fluir natural. Si el proceso es forzado, la materialización de la competencia a estimar se podría ver afectada de forma notoria. Se requiere, entonces, que estos aspectos sean cubiertos a partir de un plan de evaluación, especificando estadios y tiempos precisos en relación con los estudiantes y los docentes.

Así pues, como indica Canabal (2011), se deben utilizar estrategias holísticas, entre ellas las carpetas de aprendizaje, los estudios de casos, los proyectos colaborativos, las simulaciones, entre otras. De esta forma, se potencia una evaluación orientada al desarrollo de las competencias desde una perspectiva formativa, por tanto, dirigida a la adquisición de conocimientos teóricos y prácticos, capacidades, destrezas, aptitudes, habilidades, actitudes, a lo largo de todo el proceso de aprendizaje. En este complejo proceso se requiere el empleo de diversos procedimientos de evaluación: "no basta sólo con evaluar lo que el (la) estudiante conoce, define y recuerda, sino que también deben evaluarse sus habilidades cognitivas, lo que comprende, relaciona, integra, contrasta y transfiere" (Margalef, 2011, p. 30).

En el contexto de la FCFM, la innovación está enfocada en las metodologías docentes y en los procesos de evaluación y de retroalimentación del aprendizaje con el fin de fortalecer su efectividad y su aporte a los procesos de aseguramiento de la calidad de la formación de pregrado. Todo esto bajo el reconocimiento de la diversidad de los y las estudiantes y de los contextos en los que se forman (González, 2016). Las estrategias a desarrollar se basan en la experiencia y aprendizajes logrados por la Universidad de Nueva Gales del Sur a través del "Assessment Project" (Marshall, Henry y Ramburuth, 2013), cuyo objetivo es generar procesos más eficientes y efectivos de evaluación y retroalimentación para el aprendizaje, con un fuerte énfasis inclusivo y optimizando a la vez la carga laboral de los académicos.

\section{Aspectos metodológicos}

La plataforma metodológica general de este estudio es la investigación-acción. La investigación-acción apunta hacia la comprensión y transformación de las realidades socioeducativas. Está orientada a la emancipación de los participantes a través de una transformación profunda de las organizaciones sociales y las prácticas educativas. Incorpora la teoría crítica, se esfuerza por cambiar las formas de trabajar y enfatiza en la formación del profesorado (Colmenares y Piñero, 2008). La realidad es interpretada y transformada con miras a contribuir 
en la formación de individuos más críticos, más conscientes de sus propias realidades, posibilidades y alternativas, de su potencial creador e innovador, en definitiva, personas autorrealizadas. Por lo tanto, epistemológicamente se puede señalar que existe una dialogicidad permanente entre los grupos de investigación y las comunidades, en los que no existen jerarquías rígidas y todos los miembros son responsables de las acciones y de las transformaciones que se generen en el proceso investigativo-participativo.

Esta metodología posibilita a los docentes y asesores reconstruir e interpretar la innovación desarrollada, privilegiando los saberes y el punto de vista de los participantes. Las investigaciones de este tipo se orientan a la resolución de problemas (Freebody, 2003) y corresponde a un ciclo en espiral, producto de una serie de iteraciones en las que se aplican las innovaciones que resultan de la investigación: "Al poner a prueba estas nuevas ideas no se busca únicamente teorizar acerca de la práctica educativa o agregar conocimiento a la disciplina, sino también mejorar la práctica" (Ávila y Cortés, 2018, p. 160). Las fases del plan de investigación-acción son las siguientes: 1) diagnóstico, 2) reflexión de la práctica docente 3) la implementación de nuevas metodologías y 4) evaluación.

Fase №1 Diagnóstico: Se recoge información mediante encuestas de evaluación docente aplicadas a los estudiantes durante dos momentos, una primera instancia a mitad de semestre y la segunda a término de semestre. A esto se agrega la información recolectada a través del instrumento de Retroalimentación Estudiantil Temprana (RET) (Mancilla, Salinas y Matheson, 2017). Estos tres instrumentos indagan en la percepción que tienen los estudiantes respecto a su proceso formativo. El diagnóstico se llevó a cabo durante el segundo semestre de 2017. Los resultados globales de las siete secciones del curso fueron separados en dos grandes ámbitos: fortalezas y debilidades.

Fase $N^{`} \mathbf{2}$ Reflexión docente: Esta fase contempla el análisis de las prácticas académicas del propio equipo docente del curso. Estas reflexiones se efectuaron durante las reuniones de equipo semanal durante el segundo semestre 2017. Estas reuniones fueron integradas también por especialistas en enseñanza y evaluación, integrantes del Área para el aprendizaje de Ingeniería y Ciencias (A2IC) y por especialistas del Programa de Alfabetización
Avanzada. Las áreas de apoyo a la docencia (A2IC y Programa de Alfabetización Avanzada) se establecen como asesores(as) técnico-pedagógicos del plan de trabajo con líneas de acción determinadas: A2IC como unidad asesora del ámbito de la enseñanza y evaluación y Programa de Alfabetización como unidad encargada de propiciar la instalación de las competencias de comprensión y producción de textos orales, escritos y multimodales en el nuevo programa de curso.

Fase $N^{\circ} 3$ Implementación de nuevas metodologías: En esta etapa se diseñaron nuevos materiales didácticos y evaluativos, asimismo se consensuaron criterios entre el equipo docente. Los nuevos recursos didácticos y evaluativos se implementaron durante el primer semestre otoño 2018.

Como se observa en la figura 2, la innovación consensuada es fruto de la reflexión del equipo docente (fase 2) y del análisis de las encuestas (fase 1). Se suma a este plan, las jornadas de formación que fueron preparadas por los equipos asesores de la facultad.

En este contexto, entonces, durante este periodo de ajuste del curso (fase 3), el A2IC ejecutó dos jornadas de capacitación para los académicos: la primera sobre cómo desarrollar y evaluar la competencia de trabajo en equipo en los estudiantes, con la finalidad de mejorar los lazos de comunicación $\mathrm{y}$, al mismo tiempo, puedan adquirir la habilidad de enseñar a sus estudiantes a trabajar en equipo. La segunda tuvo énfasis en planificación, estrategias y evaluación. Además, se llevó a cabo una capacitación b-learning para los auxiliares y ayudantes del curso en el módulo de trabajo en equipo para lograr una mayor concordancia y coherencia en los procesos formativos en todos los actores (académicos, ayudantes y auxiliares).

Por su parte, el Programa de Alfabetización Avanzada asesora en la instalación de la Competencia Genérica 1 luego del levantamiento de los resultados de aprendizaje asociados a esta competencia en el programa y en el syllabus del curso en consonancia con el Modelo Educativo Institucional (UCHILE, 2018). Además, se diseña e implementa un Módulo de Comunicación Oral al interior del trabajo del curso. También, por medio de un trabajo colaborativo e interdisciplinario, se diseñan materiales didácticos específicos como guías para el diseño de un blog, para la producción de un informe o reporte técnico producido por los estudiantes, 


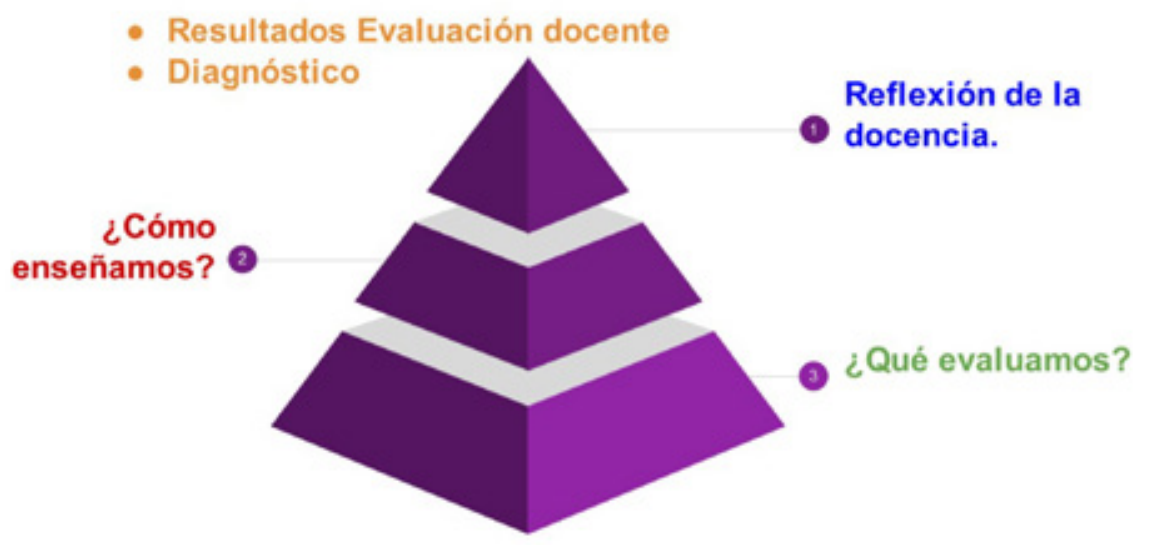

Figura 2: Plan de mejora de los procesos de enseñanza - aprendizaje

orientaciones clave para la exposición oral formal, entre otros. Este curso contó, además, con el Programa de Acompañamiento LEA UCHILE (2019). En efecto, durante el semestre los estudiantes de cada sección tuvieron alta participación de las tutorías LEA FCFM, se realizaron más de 50 tutorías en diferentes temáticas asociadas con el desarrollo del discurso escrito y oral en contextos disciplinares y académicos.

Fase $N^{\circ} \mathbf{4}$ Evaluación: En esta fase se considera en un primer momento, la realización de un levantamiento de información por medio de una evaluación de curso, dirigida a los académicos, a los auxiliares y ayudantes de curso, un grupo focal con preguntas dirigidas y tiempos de reflexión sobre cómo realizan su docencia y cómo los estudiantes los visualizan a través de la encuesta docente. En un segundo momento, se desarrolló un grupo focal y un cuestionario en línea con preguntas dirigidas y espacios de reflexión, tanto para los ayudantes como para auxiliares del curso. $Y$, en un tercer momento, se aplicó nuevamente RET. Además, el equipo docente realizó observación de clases.

\section{Instrumentos de recolección de información}

La siguiente tabla presenta la descripción de cada uno de los instrumentos utilizados en este ciclo de investigación-acción:

Tabla 3: Descripción de Instrumentos

\begin{tabular}{|l|l|}
\hline \multicolumn{1}{|c|}{ NOMBRE DEL INSTRUMENTO } & \multicolumn{1}{c|}{ DESCRIPCIÓN } \\
\hline $\begin{array}{l}\text { RET } \\
\text { (Retroalimentación Temprana a Estudiantes) }\end{array}$ & $\begin{array}{l}\text { Es una estrategia de retroalimentación formativa para el docente, su propósito es identificar } \\
\text { algunos aspectos concretos que puedan aportar a la mejora del curso que podrían incorporarse } \\
\text { en el tiempo restante del semestre. Se implementa transcurridas 4 a } 5 \text { semanas. Contempla las } \\
\text { dimensiones: desarrollo de habilidades, preparación de la enseñanza y compromiso formativo. }\end{array}$ \\
\hline Encuesta medio semestre & $\begin{array}{l}\text { Es un instrumento institucional de percepción y tiene como propósito evaluar el proceso for- } \\
\text { mativo y de esta manera proponer remediales oportunos. Se aplica a los estudiantes durante } \\
\text { mediados de semestre y está compuesta por las dimensiones: Conocimiento y dominio de las } \\
\text { materias, habilidades pedagógicas, organización del curso, diseño del curso, relaciones inter- } \\
\text { personales y evaluaciones. }\end{array}$ \\
\hline Encuesta Docente & $\begin{array}{l}\text { Corresponde a un instrumento institucional de percepción que se aplica a los estudiantes du- } \\
\text { rante el término del semestre y se compone de las dimensiones: Conocimiento y dominio de } \\
\text { las materias, habilidades pedagógicas, organización del curso, diseño del curso, relaciones } \\
\text { interpersonales y evaluaciones. }\end{array}$ \\
\hline Observación de clases & $\begin{array}{l}\text { Corresponde a una estrategia de retroalimentación docente que se implementa durante todo } \\
\text { el proceso formativo de los estudiantes. Su diseño contempla las dimensiones: preparación de } \\
\text { la enseñanza, evaluación de los aprendizajes, comunicación; gestión de problemas de aula. }\end{array}$ \\
\hline
\end{tabular}


Tabla 4: Momentos de evaluación, instrumentos y aspectos evaluados

\begin{tabular}{|l|l|l|}
\hline \multicolumn{1}{|c|}{ ASPECTOS POR EVALUAR } & \multicolumn{1}{|c|}{ INSTRUMENTO } & MOMENTO DE APLICACIÓN \\
\hline $\begin{array}{l}\text { Metodologías aplicadas } \\
\text { Enseñanza de las competencias genéricas: } \\
\text { Comunicación académica y profesional e innovación. }\end{array}$ & $\begin{array}{l}\text { RET } \\
\text { Encuesta medio semestre } \\
\text { Encuesta docente }\end{array}$ & $\begin{array}{l}\text { Primavera } 2017 \\
\text { Primavera } 2018\end{array}$ \\
\hline $\begin{array}{l}\text { Enseñanza de las competencias genéricas: } \\
\text { Comunicación académica y profesional e innovación. }\end{array}$ & $\begin{array}{l}\text { RET } \\
\text { Observación de clases }\end{array}$ & $\begin{array}{l}\text { Primavera } 2017 \\
\text { Otoño } 2018 \\
\text { Primavera } 2018\end{array}$ \\
\hline
\end{tabular}

En la Tabla 4 se pueden encontrar los aspectos evaluados, tipo de instrumento y momento de aplicación en el ciclo de investigación-acción.

\section{Resultados de investigación y acción}

La presentación de los resultados obtenidos en esta investigación se ha estructurado de acuerdo con las fases de investigación-acción:

\section{Fase 1 Diagnóstico:}

El análisis de resultados de los tres instrumentos arrojó ciertos puntos críticos en los procesos de enseñanza y de evaluación, los que deben ser fortalecidos por medio de una innovación pedagógica contextualizada. En términos de fortalezas, el estudiantado consultado valoró del curso el trabajo en equipo, la experiencia desde la práctica, la aplicación de la ética y de habilidades "blandas". En cuanto a las debilidades del curso, la consulta arrojó la percepción de escasa preparación de la enseñanza, la ausencia de cátedras activas, rigidez en el uso de metodologías y cambios en el proyecto, baja optimización de las horas de laboratorio y poca claridad en la evaluación.

\section{Fase 2 Reflexión de la práctica docente:}

Con los resultados del diagnóstico, el equipo docente del curso estableció en reuniones de trabajo una serie de preguntas (probes) para estimular la reflexión y dialogar en torno a su desempeño docente. Los principales ejes de reflexión fueron: en primer lugar, el replanteamiento de los resultados de aprendizaje del curso (¿son claros y entendibles por los estudiantes?); en segundo lugar, la revisión de las estrategias de enseñanza-aprendizaje desplegadas en el curso (ison efectivas las diversas estrategias de enseñanza utilizadas para lograr los resultados de aprendizaje declarados en el programa de curso que imparte?); en tercer lugar, las formas de evaluación (ise utilizan evaluaciones formativas y sumativas ajustadas a los objetivos del curso y a las necesidades de los estudiantes?); en cuarto lugar, el análisis de los aprendizajes efectivos que logran los estudiantes (si sus estudiantes no están logrando el aprendizaje deseado: ¿he rediseñado la forma de enseñar, he pensado en otras alternativas?).

La revisión de estos cuatro ejes permitió lograr un punto de equilibrio para la mejora de los procesos formativos. De esta manera, se generaron espacios de discusión docente y se estableció la posibilidad de apoyar la docencia con diversas estrategias de enseñanza, estrategias que integran los entornos digitales y de las nuevas tecnologías de la comunicación e información que han producido un cambio notable en la valoración del conocimiento, el manejo de la información y en las formas de aprendizaje. En este sentido, la irrupción de nuevos medios, modos, estructuras y maneras de comunicarse y resolver problemas, han generado nuevos contextos y, en consecuencia, han contribuido a generar cambios en las formas en que pensamos, escribimos, leemos (Parodi, Burdiles, Moreno-De León y Julio, 2018) y buscamos soluciones a problemas cotidianos.

Es así que profesores y estudiantes enfrentan nuevos desafíos en el contexto de la sociedad del conocimiento, en el que los dispositivos digitales involucran un despliegue de habilidades complejas de adquisición y transformación del mismo, así como el aprendizaje es a lo largo de toda la vida y no es de carácter estanco ni monolítico. En este escenario, por tanto, se requieren las habilidades de aprender a aprender y del aprendizaje para la comprensión, que implica "la capacidad de usar conocimientos, conceptos y habilidades en curso para iluminar nuevos problemas o temas no previstos" (Gardner y Boix-Mansilla, 1994, p. 14). 
Asimismo, en dicho análisis, el equipo docente percibe como una debilidad la disociación y falta de acuerdo entre el equipo de académicos. Esto se traduce en la poca efectividad del trabajo en equipo y en colaboración. Por lo que instalar el trabajo colaborativo entre los docentes del curso constituye un aspecto relevante del nuevo plan de innovación para la mejora de la docencia.

Fase 3 Implementación de nuevas metodologías (Plan de innovación para la mejora):

En esta fase, el equipo docente y el equipo de asesores técnicos diseñan los ajustes y cambios al curso con el objeto de aumentar los niveles de aprendizaje profundo y motivación por el curso. El principal objetivo es implementar estrategias de enseñanza y de evaluación que propicien la participación y motivación de los estudiantes en clases y permitan evaluar las competencias genéricas de comunicación e innovación. Para el logro de los objetivos propuestos, se rediseña el programa del curso y se establecen las metodologías docentes y los sistemas evaluativos de la nueva propuesta. De acuerdo con lo anterior, los principales recursos que se generaron para este nuevo diseño del curso fueron:

1.- Plataforma U-curso: esta plataforma es un servicio de apoyo a la docencia presencial que busca organizar el material educativo mediante la creación de sitios de aprendizajes virtuales para cada curso (para visitar UCAMPUS ir a https://www.U-cursos.cl/). Dentro de los beneficios de U-curso se encuentra el establecer estándares de calidad comunes entre las distintas unidades de la universidad, mejorar la comunicación con los estudiantes, compartir material de estudio, calcular y publicar notas, entre otros. La nueva propuesta plantea intencionar el uso de esta plataforma en los estudiantes, incorporando mensajería entre docentes y estudiantes, recordatorios en foro y publicación sistemática de materiales y recursos de consulta.
2.- Habilitación de Blog: Es un sitio web alojado en U-curso con forma de bitácora o diario personal, donde los contenidos suelen utilizarse de manera frecuente y exhibirse en orden cronológico: los lectores o visitadores del blog tienen la posibilidad de realizar comentarios sobre lo publicado.

3.- Habilitación de Foro: Espacio virtual de la plataforma de U-curso donde se reúnen un determinado grupo de personas para intercambiar temas, experiencias o ideas en común.

4.- Evaluación de avance mediante infografías: Es un texto con apoyo de imágenes que se caracteriza por transmitir información resumida de un proyecto, investigación y/otros. Tiende a ser breve.

5.- Vídeos de clase invertida (Flipped classroom): Se trata de una herramienta didáctica en la que la exposición de contenido se hace por medio de videos o recursos educativos que pueden ser consultados en línea de manera libre, mientras el tiempo de aula se dedica a la discusión, resolución de problemas y actividades prácticas bajo la supervisión y asesoría del profesor. Estos vídeos fueron subidos a la plataforma U-Curso.

\section{6.- Evaluación de avance mediante exposicio- nes orales con apoyo TIC (PPT): Los estudiantes} explican con apoyo visual ante un auditorio formado por sus pares, docentes, ayudantes y auxiliares los principales hallazgos y características de sus proyectos de innovación.

De esta manera, a través de estos seis recursos se fortalecieron las metodologías innovadoras de enseñanza y los procesos evaluativos del curso de primer año denominado 'Introducción a la Ingeniería', impactando a 840 estudiantes de nuevo ingreso. Asimismo, se incorporó mediante esta metodología la enseñanza explícita de géneros discursivos de formación académica o epistémicos (Thaiss y Zawacki, 2006).
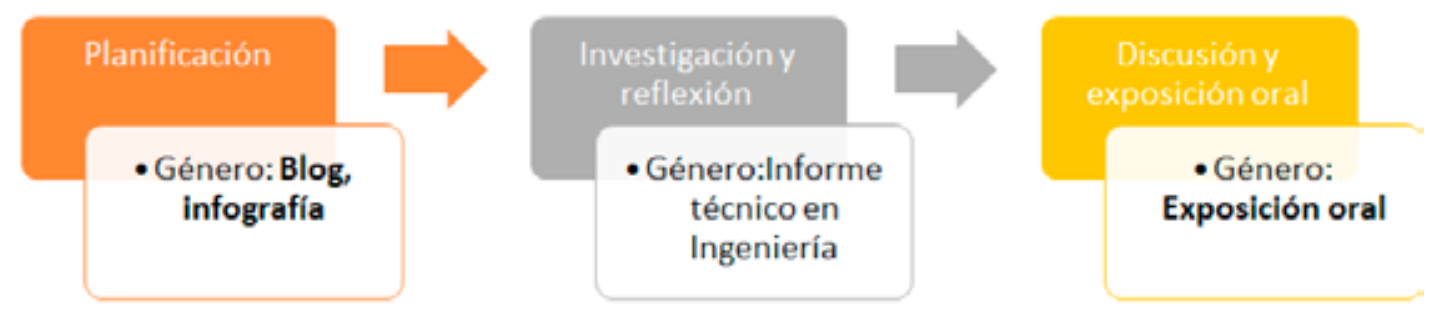

Figura 3: Evaluación de aprendizajes por medio de la comunicación escrita: Cadena de géneros discursivos (Marinkovich, Sologuren y Shawky, 2018) y progresión de la competencia comunicativa CG1 (FCFM, 2018) 


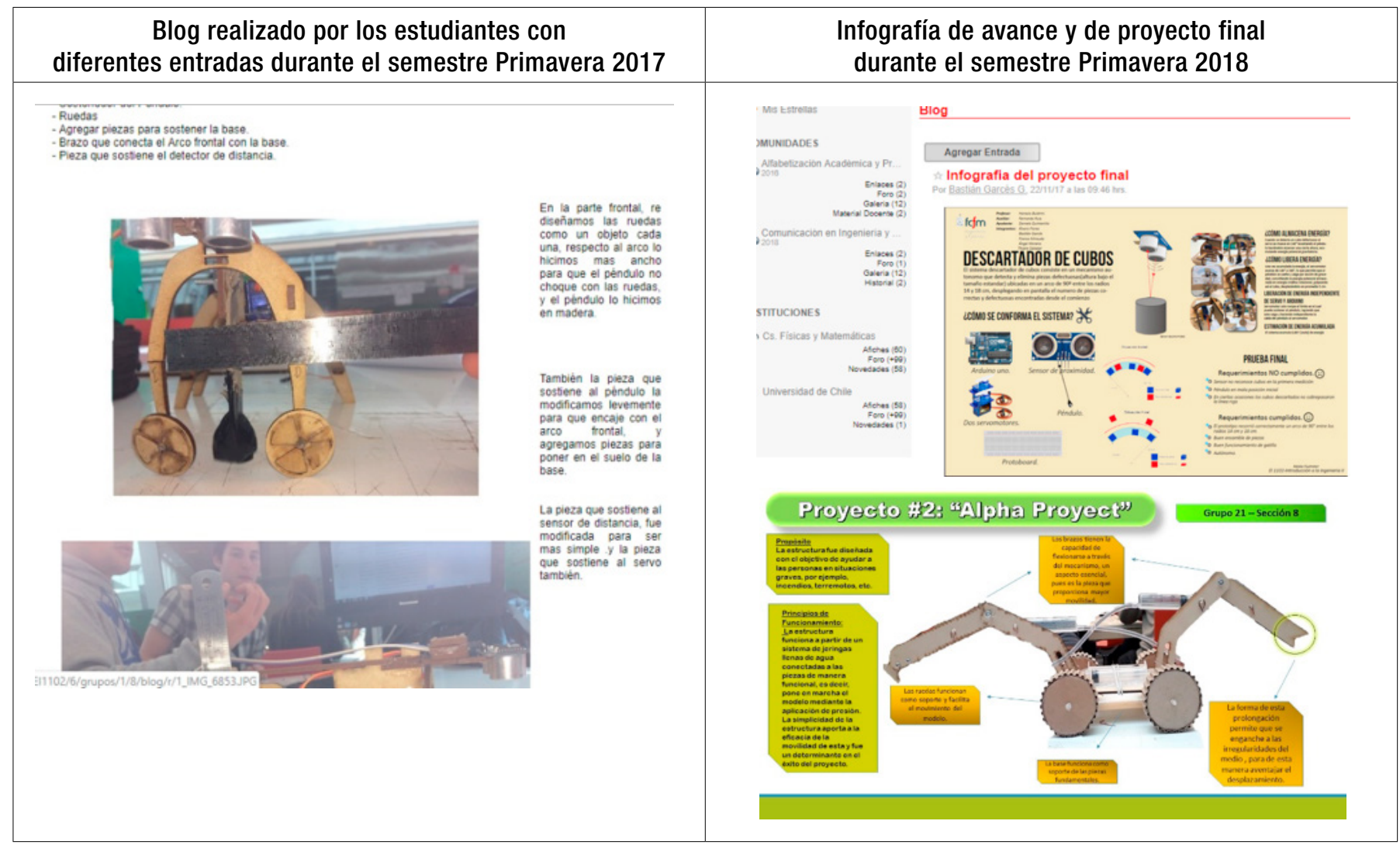

Figura 4: Recursos didácticos para enriquecer la enseñanza de la ingeniería.

\section{Antes}

Estudio de Caso: Con una única solución

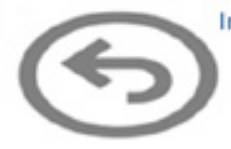

PPT
Informe

Exposiciones orales

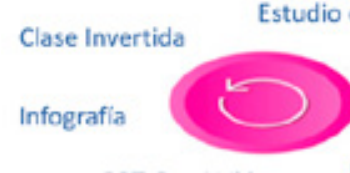

PPT, Prezi Videos

\section{Después}

\author{
Modelamiento
}

Figura 5: Estrategias de enseñanza-aprendizaje en las dos versiones del curso

Así, en el proceso de planificación, los estudiantes elaboraron blogs e infografías que les permiten sistematizar y visualizar de forma más clara los avances de su proyecto o desafío de ingeniería. En la Figura 4 es posible apreciar un ejemplar de cada uno de estos géneros discursivos producidos por los equipos de trabajo, equipos organizados en torno a un proyecto común.

De esta forma, cada uno de estos recursos de aprendizaje elaborados y compartidos en la plataforma U-curso (UCAMPUS) potenciaron un aprendizaje activo, ya que el desarrollo de la comunicación y de la innovación se desarrolló a partir de las exigencias de cada uno de los desafíos de ingeniería y ciencias, configurándose así una literacidad en innovación y una literacidad para la innovación, en el marco de las habilidades que son necesarias para enfrentar el siglo XXI.

La Figura 5 nos permite visualizar las nuevas metodologías didácticas y evaluativas que fueron incorporadas a partir de los procesos de revisión y reflexión docente. 
Fase 4 Evaluación: Dentro de los resultados obtenidos se encuentran: Primero, la encuesta docente de mitad de semestre de primavera 2017 y la encuesta docente de mitad de semestre de primavera 2018. La comparación entre ambas revela un significativo aumento de las percepciones positivas en el alumnado en relación con las nuevas metodologías aplicadas en el curso, especialmente, destaca el proceso de retroalimentación aplicado. En efecto, la calificación global de desempeño aumenta considerablemente, esta calificación fluctúa de 1 a 7 puntos (tres de cinco académicos se encuentran entre 4,95 a 5,8 y los dos restantes se encuentran entre 6,4 a 6,9). Segundo, las encuestas docentes de final de semestre (primavera 2017 y primavera 2018) arrojan resultados similares (tres de los cinco académicos se encuentran entre 5,51 a 5,8 y los dos restantes se encuentran entre 6,4 a $6,7)$. La comparación entre ambas revela una mejor percepción por parte del estudiantado sobre el desempeño de los docentes del curso.

Tercero, al comparar los resultados de la RET Primavera 2017 y Primavera 2018, se observa que en la RET aplicada durante Primavera 2018, los estudiantes consignan un aumento en el fortalecimiento del trabajo en equipo, el trabajo en colaboración, el desarrollo de la creatividad y de la investigación por medio de las soluciones a problemas cotidianos. En relación con los nudos críticos en la metodología de enseñanza-aprendizaje, la percepción de los estudiantes se focaliza en establecer con claridad el propósito de las clases, cómo serán evaluados, presencia de ejemplos prácticos, cátedras más didácticas y fortalecer los procesos de aprendizaje por medio de la retroalimentación. En síntesis, se evidencia que la percepción de los estudiantes en relación con la forma de enseñar y evaluar el proceso de enseñanza-aprendizaje aumentó entre 2,0 a 3,0 puntos en dos casos como se puede apreciar en la Figura 6.

En la Figura 6 se observa un alto porcentaje de percepción positiva por parte del estudiantado en relación con la implementación de nuevas estrategias de enseñanza y de procedimientos evaluativos, valorando positivamente el trabajo realizado.

La Figura 7 consigna un aumento en la percepción positiva por parte de los estudiantes en relación con el trabajo del profesor en el desarrollo y evaluación de la competencia de comunicación (CG1) y en la competencia de innovación (CG6).

Finalmente, en la Figura 8 es posible apreciar el proceso de evaluación de las competencias genéricas

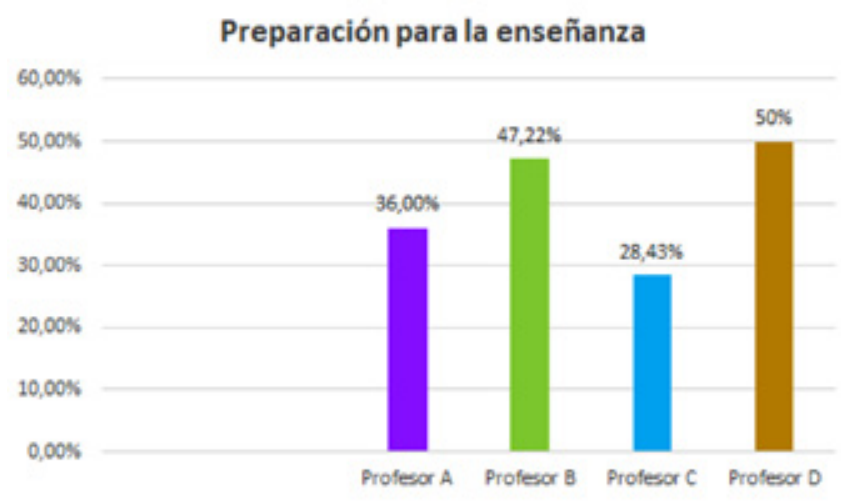

Figura 6: Percepción de los/as estudiantes en torno a la implementación de nuevas metodologías y procedimientos de evaluación
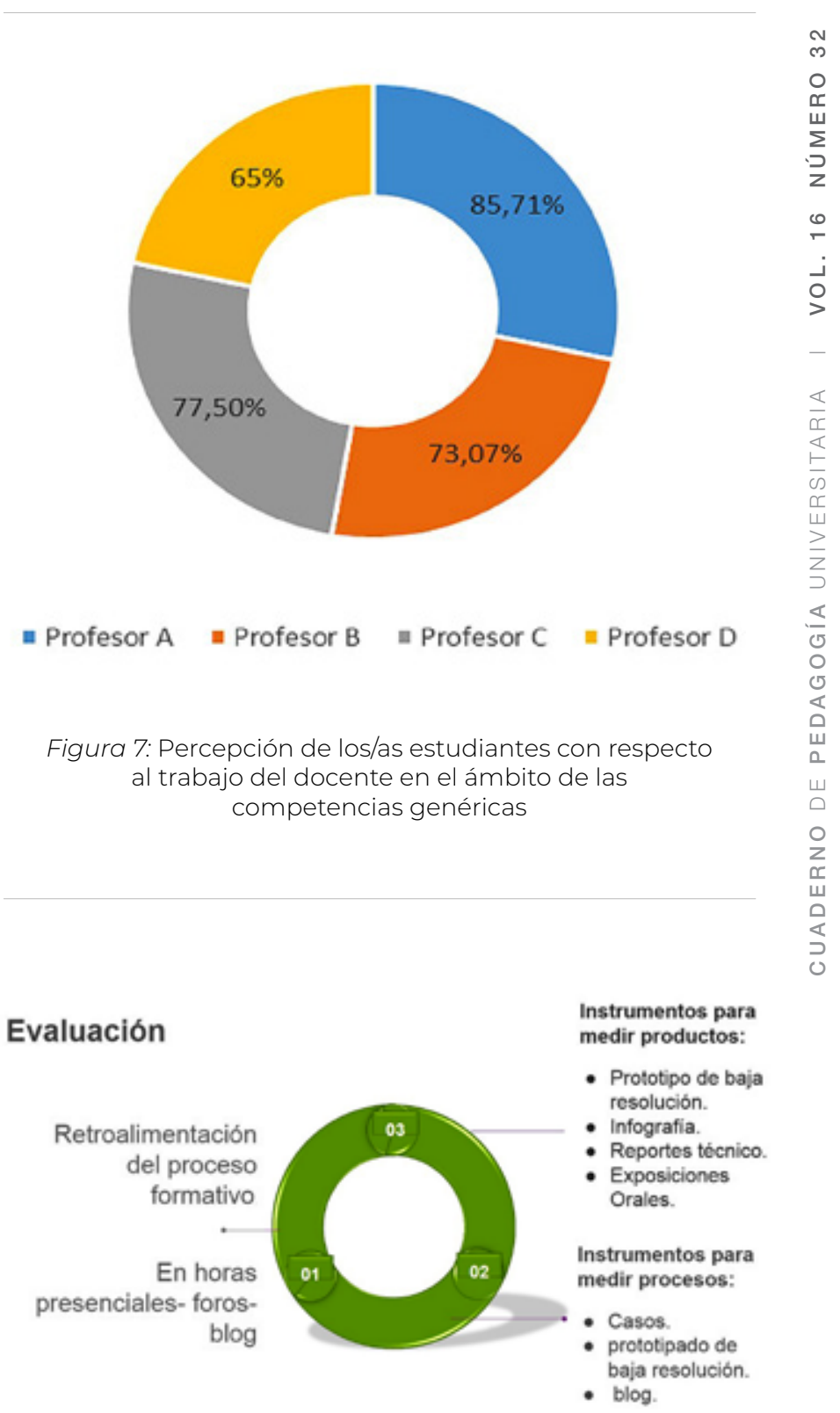

Figura 8: Proceso de Evaluación de las competencias genéricas del curso Introducción a la Ingeniería 
en el espacio curricular de introducción a la ingeniería en el ciclo inicial de la formación de pregrado. En este proceso se consideró la evaluación tanto del proceso como del producto.

De forma complementaria a la aplicación de los instrumentos descritos, se revisaron los foros del curso y los e-mails de la plataforma U-curso en el semestre Primavera 2017 y 2018 y se constató un aumento en el porcentaje de consultas a través de estos medios de comunicación (los estudiantes desarrollaron consultas a sus académicos, ayudantes, auxiliares y resolvieron los desafíos planteados), lo que significa un grado de satisfacción del uso de estos recursos como un medio para fortalecer la comunicación efectiva ante las dudas emergentes a desafíos propuestos en el curso.

\section{Reflexiones finales}

En este trabajo nos propusimos responder la siguiente pregunta de investigación: ¿Cómo contribuir hacia un modelo de aprendizaje activo del curso de Introducción a la Ingeniería que fortalezca la formación en competencias genéricas? Pregunta que impulsó un ciclo de investigación-acción cuyo objetivo fue el diseño de una propuesta de estrategias y recursos de aprendizaje activo y su evaluación para el desarrollo de competencias genéricas en los primeros años de ingeniería y ciencias. Este set de instrumentos se construyó gracias al trabajo colaborativo e interdisciplinario entre ingenieros-docentes de la facultad y las unidades de apoyo a la docencia y al aprendizaje en el área de STEM.

Los principales resultados de esta investigación-acción muestran un aumento significativo en la satisfacción positiva por parte de los estudiantes en relación con la implementación de nuevas estrategias de enseñanza y de procedimientos evaluativos (Encuesta docente de mitad de semestre, Encuesta Docente Final y RET). Asimismo, en la instalación de nuevas metodologías para las competencias genéricas de Comunicación e Innovación, el curso trabajado se convirtió en un espacio formativo clave para el desarrollo de dichas competencias al interior del Plan Común de Ingeniería y Ciencias. En este sentido, más de 800 estudiantes de nuevo ingreso 2018 fueron impactados.

Luego de la evaluación de este plan de mejoramiento innovado, se sostiene la importancia de la indagación de nuevas formas de enseñanza por parte del profesorado, un tránsito a metodologías activas de enseñanza y aprendizaje en educación superior. Así como la necesidad de los cuerpos docentes de implementar la reflexión en sus espacios de trabajo docente, espacios en los que la investigación de su propia actuación se vuelve esencial. En este mismo sentido, se hace imperativo que los docentes busquen el apoyo de los asesores técnicos de la institución para obtener espacios de formación de los cuerpos docentes. De esta forma, el trabajo colaborativo e interdisciplinario transforma la docencia, generando un impacto significativo en la forma de aprender de los estudiantes.

Además, es importante destacar que mediante este ciclo de investigación-acción se instala una cultura de reflexión profunda de las prácticas académicas por parte de cada uno de los docentes. En efecto, se establecieron y relevaron los aspectos a fortalecer, dentro de estos la comunicación al interior del equipo. Esta reflexión instala reuniones de trabajo sistemáticas y colaborativas. Además, al finalizar el año académico 2018, la experiencia del estudio fue presentada en una de las mesas de trabajo del Consejo de Escuela de la facultad donde se socializó a otros académicos y departamentos lo que permitió dar un cierre a la primera espiral de investigación-acción. Por lo que dentro de este ciclo se ejecutaron diversas acciones (metodológicas y evaluativas) y nuevos recursos, metodologías de enseñanza, competencias de investigación y de aprendizaje a través de la acción, tales como: el uso de plataforma $U$-curso, el blog, la infografía, los vídeos como recurso de clase invertida, las exposiciones orales con apoyo TIC (PPT).

Se concluye que el proceso analítico-reflexivo que se ha llevado a cabo en el interior del cuerpo docente ha proporcionado el replanteamiento tanto del macrocurriculum (los programas de formación de pregrado) y el microcurriculum (el aula universitaria) de una manera completamente distinta, lo que nos ha dado una oportunidad única para desarrollar procesos de investigación-acción y de desarrollo de nuevos recursos didácticos adaptados a la necesidades locales y a la identidad de disciplinas y carreras con una alta heterogeneidad cultural.

En una línea más cualitativa, se destaca el aporte de esta experiencia de asesoramiento técnico-pedagógico en el cuerpo docente dado el aumento de la reflexión sobre la práctica académica que se instaló en las reuniones de trabajo semanal a través de un cuestionamiento continuo sobre cómo mejorar los procesos de aprendizaje y de evaluación para los estudiantes. Además, la alta conciencia de que todos 
aprendemos de distinta manera, de la diversidad, de los estilos de aprendizaje y de la inclusión que están presentes en nuestras aulas, de mirar y remirar nuestras formas de evaluar, si estamos evaluando cómo enseñamos y qué estamos haciendo para subsanar los aprendizajes no logrados y cómo son los procesos de retroalimentación. En este sentido, una de las proyecciones del artículo se relaciona con el levantamiento de un sistema de evaluación de las competencias genéricas y de seguimiento de hitos evaluativos a través del curriculum.

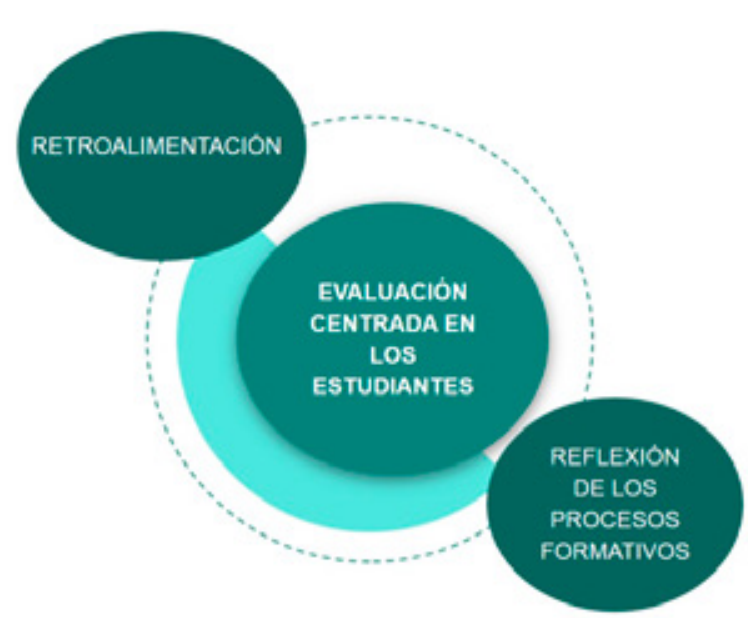

Figura 9: Proceso de evaluación en el curso de Introducción a la Ingeniería

Por lo anterior, se destaca el surgimiento de una cultura evaluativa en el seno de este equipo de profesores, una cultura de reflexión sobre las prácticas educativas, la importancia de instalar nuevas estrategias de enseñanza-aprendizaje centradas en el estudiante. Todo esto contribuye a fortalecer el aseguramiento de la calidad en los procesos formativos y su alineamiento constructivo (Biggs, 2005).

Finalmente, esta experiencia otorga insumos incipientes para desarrollar nuevas investigaciones en las temáticas de trabajo en colaboración con el equipo docente del curso, reflexión de las prácticas académicas, estrategias de enseñanza-aprendizaje y monitoreo del proceso de desarrollo de las competencias genéricas de comunicación e innovación.

\section{Referencias bibliográficas}

Ahumada, P. (2005). La evaluación auténtica: un sistema para la obtención de evidencias y vivencias de los aprendizajes. Perspectiva Educacional Formación de Profesores, 45, 11-24. Recuperado de: http://wnw.redalyc.org/articulo. oa?id=333329100002
Arnold, R. (2002). Formación profesional: nuevas tendencias y perspectivas Montevideo, Uruguay: CINTERFOR.

Ávila, N., y Cortés, A. (2018). El género 'Informe de caso' en la formación inicial docente: Una aproximación basada en la actividad. Lenguas Modernas, (50), 153 - 174.

Beneitone, P., Esquetini, C., González, J., Maletá, M. M., Siufi, G. y Wagenaar, R. (Eds.). (2007). Tuning América Latina. Reflexiones y perspectivas de la educación superior en América Latina. Informa Final. Deusto, España: Universidad de Deusto publicaciones.

Biggs, J. (2005). Calidad del aprendizaje universitario. Madrid: Narcea Ediciones.

Breeze, R., y Sancho Guinda, C. (2017). Genre-based strategies for integrating critical and creative thinking in engineering and journalism. ESP todayjournal of English for Specific Purposes at tertiary level, 5(2), 196-221.

Brown, S. (2015). La evaluación auténtica: el uso de la evaluación para ayudar a los estudiantes a aprender. Relieve, 21(2), 1-10. Recuperado de: https://ojs. uv.es/index.php/RELIEVE/article/view/7674/7464

Camilloni, A. R. W. de (2009). Estándares, evaluación y currículo. Archivos de Ciencias de la Educación, 3(3), $55-68$

Canabal, C. (2011). Paradojas y dilemas en la evaluación de competencia. En F. Labrador y R. Santero (coords.). Evaluación global de los resultados del aprendizaje en las titulaciones dentro del Espacio Europeo de Educación Superior (pp. 79-93). Madrid: Dykinson.

Coll, C., Rochera, M., Mayordomo, R. y Naranjo, M. (2011). La evaluación continuada como instrumento para el ajuste de la ayuda pedagógica y la enseñanza de competencias de autorregulación. Cuaderno De Pedagogía Universitaria, 8(15), 14-20.

Colmenares, A. y Piñero, M. (2008). La investigación acción. Una herramienta metodológica heurística para la comprensión y transformación de realidades y prácticas socioe-ducativas. Laurus, 14(27), 96-114.

Contreras, M. E. (1995). Formulación y evaluación de proyectos. Bogotá: Unad

Erdogan, N., Corlu, M., y Capraro, R. M. (2013). Defining innovation literacy: Do robotics programs help students develop innovation literacy skills? International Online Journal of Educational Sciences, 5(1). 1-9.

Facultad de Ciencias Físicas y Matemáticas (FCFM) (2018). Nuevos planes de estudios. Escuela de Ingeniería y Ciencias. Santiago: Universidad de Chile. 
Freebody, P. (2003). Qualitative research in education: Interaction and practice. London: Sage.

Gardner, H. y Boix-Mansilla, V. (1994). Teaching for understanding-Within and across the disciplines. Educational Leadership, 51(5), 14 - 18.

González, M. I. (2016). Evaluación de la actividad docente universitaria en el contexto chileno. (Tesis Doctoral inédita). Universidad de Alcalá, Madrid, España.

Imbernón, F. (2012). La investigación sobre y con el profesorado. La repercusión en la formación del profesorado: ¿cómo se investiga? Revista electrónica de investigación educativa, 14(2), 1-9. Recuperado de http://www.scielo.org. $\mathrm{mx}$ /scielo.php?script=sci_arttext\&pid=S160740412012000200001\&lang=en

Le Boterf, G. (2011). Ingénierie et évaluation des compétences (6a ed.). Paris: Editions d'Organisation.

Lopera, M. A. y Restrepo, G. (2015). CDIO: una gran estrategia de formación en ingeniería. Ingeniería \& Sociedad, 9, 33-39.

Mancilla, R., Salinas, P. y Matheson, C. (2017). Retroalimentación estudiantil temprana: Una estrategia para enriquecer la evaluación de la docencia. Recuperado de: http://escuela.ingenieria.uchile.cl/dam/ jcr:4a084790-5416-46c7-a5e8-f2ddb018c7fe/ retroalimentacion-estudiantil-temprana-unaestrategia-para-enriquecer-la-evaluacion-de-ladocencia-sochedi-2016.pdf

Margalef, L. (2005). Los retos de la evaluación auténtica en la enseñanza universitaria: coherencia epistemológica y metodológica. Perspectiva Educacional, 45, 25-44.

Margalef, L. (2011). Pautas para la planificación docente: plan didáctico de una asignatura. Alcalá: Universidad de Alcalá.

Margalef, L. y Canabal, C. (2010). Innovar en la enseñanza universitaria. Madrid: Biblioteca Nueva.

Marshall, S., Henry, R. y Ramburuth, P. (Eds.). (2013). Improving assessment in higher education: $A$ whole of institution approach. New South Wales: UNSW Press.

Marinkovich, J., Sologuren, E. y Shawky, M. (2018). The process of academic literacy in Civil Engineering Computer Science. An approach to academic writing and its genres in a learning community. Círculo de lingüística aplicada a la comunicación, 74, 195-220.

Michavila, F. (2009). La innovación educativa. Oportunidades y barreras. Arbor: Ciencia, Pensamiento y Cultura, 185, 3-8.
Parodi, G.; Burdiles, G.; Moreno-De León, T. y Julio, C. (2018). Hábitos lectores y géneros del discurso en filosofía y en economía y negocios: del discurso académico al discurso profesional. RLA. Revista de Lingüística Aplicada, 56 (2), 117-152.

Perrenoud, P. (2003). Nouvelles identités professionnelles: de quelques enjeux de formation. En J. Astolfi (Dir.), Éducation et formation: nouvelles questions, nouveaux métiers (pp. 311342). Paris: ESF.

Perrenoud, P. (2007). Pedagogía diferenciada: de las intenciones a la acción. Madrid: Popular.

Programa de Lectura y Escritura Académica (LEA) (2019). Mi aprendizaje. Recuperado de http://www.miaprendizaje.uchile.cl/lateral_lea. php?s=lea

Roca, E. (2013). La evaluación diagnóstica de las competencias básicas. Madrid: Síntesis.

Thaiss, C. y Zawacki, T. (2006). Engaged Writers and Dynamic Disciplines: Research on the Academic Writing Life. Portsmouth, NH: Boynton/Cook, Heinemann.

Tapella, E. y Rodríguez, P. (2014). Sistematización de experiencias: Una metodología para evaluar intervenciones de desarrollo. Revista de Evaluación de Programas y Políticas Públicas, 3, 80-116.

Universidad de Chile (2018). Modelo educativo de la Universidad de Chile. Departamento de pregrado. Vicerrectoría de Asuntos Académicos Universidad de Chile. Recuperado de: http://www.libros. uchile.cl/717

Universidad Católica de la Santísima Concepción. (2014). CDIO: Una nueva visión para la educación en ingeniería. Recuperado de http://www.cdio.cl/ colio-a-new-vision-for-engineering-education.html

Uribe, R., Sologuren, E. y Matamoros, A. (2016). Instalación e implementación de las competencias comunicativas en la Facultad de Ciencias Físicas y Matemáticas de la Universidad de Chile. En XXIX Congreso Chileno de Educación en Ingeniería SOCHEDI. Pucon, Chile: Universidad de la Frontera.

Wehlburg, C. (2014). Prácticas de evaluación transformadora en la educación superior. En J. M. Garrido, A. Arenas \& D. Contreras (Eds.). Mejorando las prácticas de evaluación de los aprendizajes en la docencia universitaria. Análisis y experiencias (pp. 37- 48). Valparaíso: Ediciones Universitarias de Valparaíso.

Zabalza, M. A. (2014). La práctica reflexiva: Bases, modelos e instrumentos. Madrid: Narcea. 